
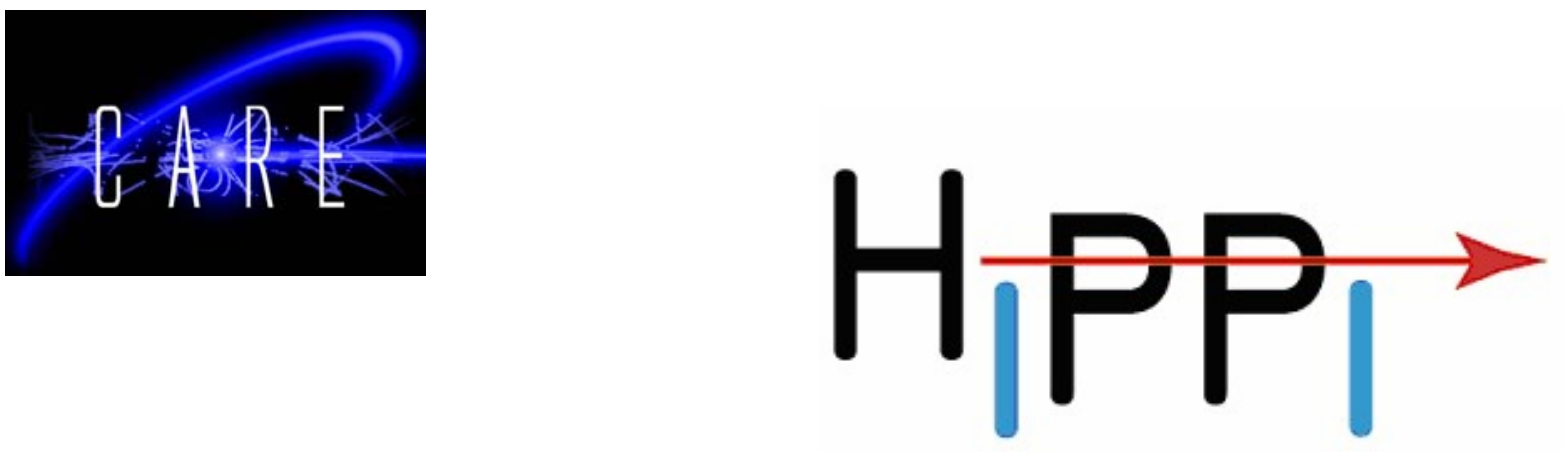

\title{
SUPERCONDUCTING RF ACCELERATING CAVITY STRUCTURAL ANALYSES
}

\author{
E. Zaplatin, Research Center Juelich, Germany
}

\begin{abstract}
Superconducting RF cavities are extensively used in particle accelerators to provide a longitudinal electric field for accelerating beam from its injection to its final energy. The power dissipation and surface field distributions need to be known so that proper provision can be made for cooling the cavity and avoiding excessively high surface fields which could result in multipactoring or quenching of superconducting cavities. As demands on performance of accelerator have increased, so have the concerns of the cavity designer with respect to the cavity mechanical properties within its cryo-environments. For most practical cavities the geometry is sufficiently complicated that analytical solutions for the electromagnetic field distributions and characteristics of the modes supported by the cavity do not exist. Prototype cavities may be built and the modes measured in the laboratory to provide the necessary information, but this would be a laborious and costly process, and gives no guarantee of finding all modes of interest to the designer. Fortunately, approximation by numerical methods enables rapid calculation and optimization of the electromagnetic fields of cavities with arbitrary-shaped structures, while ensuring that all modes of interest are found. Here we discuss different problems of SC cavity and its surrounding stand design using a series of numerical codes and methods of data exchange between them. The comparisons of numerical simulations with some experimental results are shown.
\end{abstract}

Contribution to the $8^{\text {th }}$ ICCST, Las Palmas de Gran Canaria, Spain

Work supported by the European Community-Research Infrastructure Activity under the FP6 "Structuring the European Research Area" programme (CARE, contract number RII3-CT2003-506395). 


\title{
Superconducting RF accelerating cavity structural analyses
}

\author{
E. Zaplatin \\ Research Center Juelich, Germany
}

Keywords: superconductivity, cavity, structural, electrodynamics, accelerator, fem.

\begin{abstract}
Superconducting RF cavities are extensively used in particle accelerators to provide a longitudinal electric field for accelerating beam from its injection to its final energy. The power dissipation and surface field distributions need to be known so that proper provision can be made for cooling the cavity and avoiding excessively high surface fields which could result in multipactoring or quenching of superconducting cavities. As demands on performance of accelerator have increased, so have the concerns of the cavity designer with respect to the cavity mechanical properties within its cryo-environments. For most practical cavities the geometry is sufficiently complicated that analytical solutions for the electromagnetic field distributions and characteristics of the modes supported by the cavity do not exist. Prototype cavities may be built and the modes measured in the laboratory to provide the necessary information, but this would be a laborious and costly process, and gives no guarantee of finding all modes of interest to the designer. Fortunately, approximation by numerical methods enables rapid calculation and optimization of the electromagnetic fields of cavities with arbitrary-shaped structures, while ensuring that all modes of interest are found. Here we discuss different problems of SC cavity and its surrounding stand design using a series of numerical codes and methods of data exchange between them. The comparisons of numerical simulations with some experimental results are shown.
\end{abstract}

Keywords: superconductivity, cavity, structural, electrodynamics, accelerator, fem

\section{Introduction}

The main working conditions of the SC cavities are as follow:

- Very high electromagnetic fields - maximum magnetic field on the inner cavity surface up to $B_{p k}=100 \mathrm{mT}$, maximum electric field on the inner cavity surface up 
to $E_{p k}=50 \mathrm{MV} / \mathrm{m}$. These high field result in the strong Lorenz forces which cause the cavity wall deformation;

- Low temperature - down to $2 \mathrm{~K}$, that again causes wall displacements and inner volume change after cool down;

- The pulse regime of operation that results in the addition requirements on cavity rigidity;

- High vacuum conditions $\left(10^{-9}-10^{-10}\right)$ and extra pressure on cavity walls from the helium tank also deform the cavity shape;

- High tolerances and quality surface requirements.

The deformations caused by all above mentioned reasons result in the working RF frequency shift in the range of hundreds $\mathrm{kHz}$. Taking into account high Q-factor of SC cavities such big frequency shift brings cavity out of operation. From the other hand, the use of any external tuning elements like plungers or trimmers is problematic as it results in the low down cavity acceleration efficiency. Fig. 1 shows the so-called elliptic SC cavity in its test cryostat and schematic inside view of the cavity.

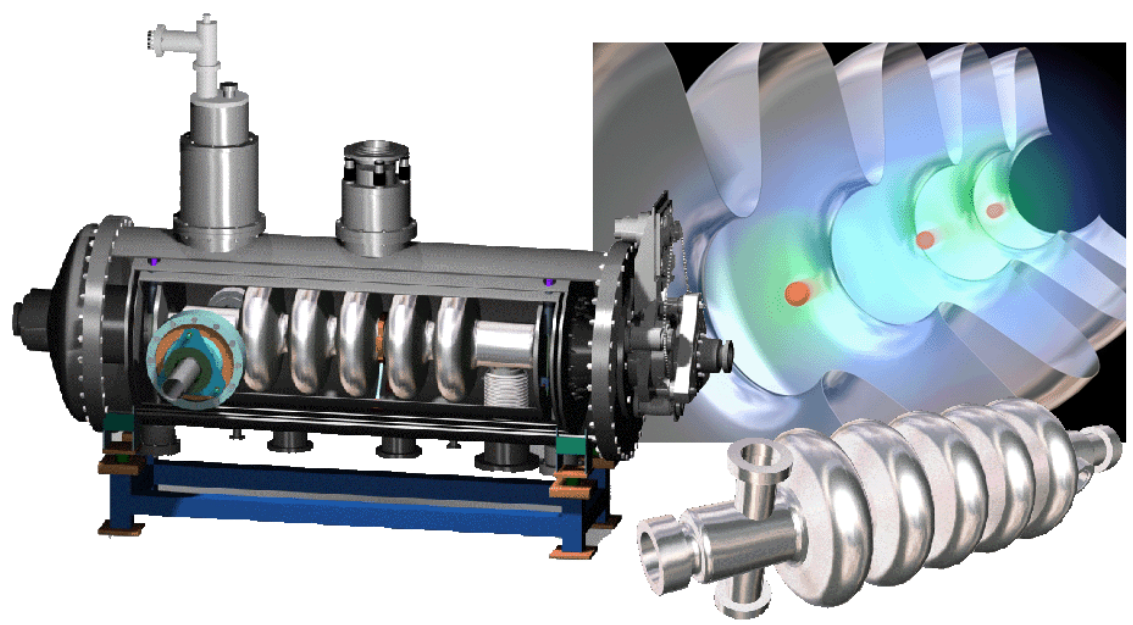

Figure 1: Superconducting elliptic cavity

The simulation model for electrodynamics is the cavity inner volume. For the system structural analysis the simulation model is the cavity walls together with cryo-module environments that could be simulated with shell and/or volume elements. It means that the different types of multi-physics applications where different physical phenomena are involved such as structural static and dynamic, thermal, fluid and high frequency electromagnetic analysis with their co-interactions should be provided during any real cavity design. The most important issue during such kind of calculations is the ability to exchange results from one type of modelling to another using them in this case as the input data and back with socalled coupled analysis.

\section{Cavity design fundamentals}


An important figure of merit for accelerating cavities is the quality factor (Q), which is proportional to the energy stored in the cavity and inverse proportional to the power dissipated in the cavity walls $\left(\mathrm{Q}=2 \pi \mathrm{f}_{0} \mathrm{U} / \mathrm{P}\right.$, where $\mathrm{f}_{0}$ is the cavity resonance frequency). The finite cavity $\mathrm{Q}$ causes the resonance to be broadened in the frequency domain. In steady state all power getting into the cavity is dissipated in cavity loss mechanisms. The frequency dependence is one way of $\mathrm{Q}$ definition. The full width at half maximum of the resonance is $1 / \tau_{\mathrm{L}}$. Another way of expressing this is that $\mathrm{Q}=\omega /(2 \Delta \omega)$. (Here $\Delta \omega$ is half the resonant width). For a typical niobium superconducting cavity operating at a frequency of $1.3 \mathrm{GHz}$ and a temperature of 1.5 $\mathrm{K}, \mathrm{Q}=1 \times 10^{10}$. With $\beta=1,2 \Delta \omega$ is only $2 \mathrm{~Hz}$, which is only $1.5 \times 10^{-7} \%$ of the frequency. In normal conducting cavities it is a few $\mathrm{kHz}$. This applies much higher requirements on SC cavity design and manufacturing.

Following issues are generally considered in design of SRF cavity:

- Minimize the peak surface fields,

- Reasonable inter-cell coupling,

- Provide required Qex,

- Reasonable mechanical stiffness,

- Safe from multipacting,

- Verification of HOM and related issues.

Many of above are coupled field problems of RF, mechanical, thermal, etc. analysis, which ask strong interfaces between simulation codes and also close cooperation between relevant areas.

The RF cavity design starts with solving electrodynamics' problem. There is a scope of codes specializing for cavity simulations (for instance, MAFIA, MWS [3], HFSS [2]). These programs are aimed for the cavity design allowing rather quick geometry change. The powerful pre-processor and existence of the cavity shape parameterization make these codes especially convenient for the iterative process of cavity optimization. As a rule, these programs can solve both types of electromagnetic problems - frequency domain and time domain.

The choice of frequency domain or time domain depends on whether separate solutions of particular resonant modes, or a time history of the excitation of the cavity, are required. A Fourier transformation allows some interchange between time and frequency domain, but is generally inefficient. Therefore, both time and frequency domain computations are used to describe the properties of an accelerator cavity. The cavity structural analysis follows the electrodynamics' design. Often a solid model of the desired cavity geometry will exist, and it is usually faster to import this geometry into ANSYS [1], rather than creating the geometry internally. Some geometry manipulation within the Finite Element Analysis model is still generally required. That's why this procedure is justified only for the calculations of the final cavity geometry. For the real iterative design work it would be rather time consuming in total and it is reasonable to build the separate model for structural analysis. The full cavity shape parameterization is highly required for both electromagnetic and mechanics.

The accelerating field, $E_{a c c}$, is proportional to the peak electric $\left(E_{p k}\right)$ as well as magnetic field $\left(\mathrm{H}_{\mathrm{pk}}\right)$ on the surface of the cavity. Therefore, the main fundamental 
aspects of superconducting cavities are the maximum surface fields that can be tolerated without increasing the microwave surface resistance substantially or without causing a catastrophic breakdown of superconductivity. The ultimate limit to accelerating field is the theoretical RF critical magnetic field. For the most popular superconductor, niobium, this is about 0.23 Tesla. Typical cavity performance is significantly below the theoretically expected surface field. One important phenomenon that limits the achievable RF magnetic field is „thermal breakdown" of superconductivity, originating at sub millimeter size regions of high RF loss, called defects. When the temperature outside the defect exceeds the superconducting transition temperature, the losses increase, as large regions become normal conducting.

In contrast to the magnetic field limit, the theoretical limit to the tolerable surface electric fields is unknown. However, at high electric fields, an important limitation to the performance of superconducting cavities arises from the emission of electrons from high electric field regions of the cavity. Power is absorbed by the electrons and deposited as heat upon impact with the cavity walls. If the emission grows intense at high electric fields it can even initiate thermal breakdown. When designing the cavity for a certain application, the shape and size will often go through many iterations before a satisfying design is found. Since the ratios of $E_{p k} / E_{a c c}$ and $B_{p k} / E_{a c c}$ determine the maximum accelerating field that can be achieved in a cavity they are important figures of merit to compare different designs and to identify the superior shape.

\section{Model meshing}

Some additional features of the specialized RF codes, like MAFIA, MWS, HFSS [12] (for instance a number of specific macros), favour to use these programs for cavity RF parameter optimisations. On the other hand, the existence of the RF code in ANSYS [3] together with ability to use the same meshed model and exchange the results between different types of simulations promises the better final results. It means the main task of electrodynamics' simulation in ANSYS is to receive the results as close as possible to the specialized RF codes. Since the further simulations of the structure will be related and normalized on the peak fields found, the solution of the proper field distribution on the surface is the first concern of this task.

An acceptable mesh creation is an iterative process. All simulation results including electromagnetic, surface heat flux, etc. are highly dependent on the mesh density. That's why the usual procedure is to create a fine mesh in critical areas on the surfaces, while retaining a larger mesh in not so important places of body in order to reduce run time and memory usage. A simple way to achieve this mesh variation is to divide the vacuum volume into sub-volumes depending on the needed local mesh size. In this way, not only the surface mesh can be controlled by sizing areas and lines but the „global“ mesh size can be set on the local basis for each sub-volume, resulting in better mesh control.

The use of regular mesh looks favourable for the axisymmetric structures. At the same time during cavity design together with its environments it is not always 
possible to provide 2D simulations. Again, the most important feature of the mesh adjustment is to control $\mathrm{E}_{\mathrm{p} k} / \mathrm{E}_{\mathrm{acc}}$ and $\mathrm{B}_{\mathrm{p} k} / \mathrm{E}_{\mathrm{acc}}$ ratio values. The presented plots (Fig.2) show that it is not obvious that the more tense mesh corresponds to the better results. Even more, for such structures the regular mesh might be disadvantageous while the axisymmetric mesh lines will cause inhomogeneous of the field distribution.
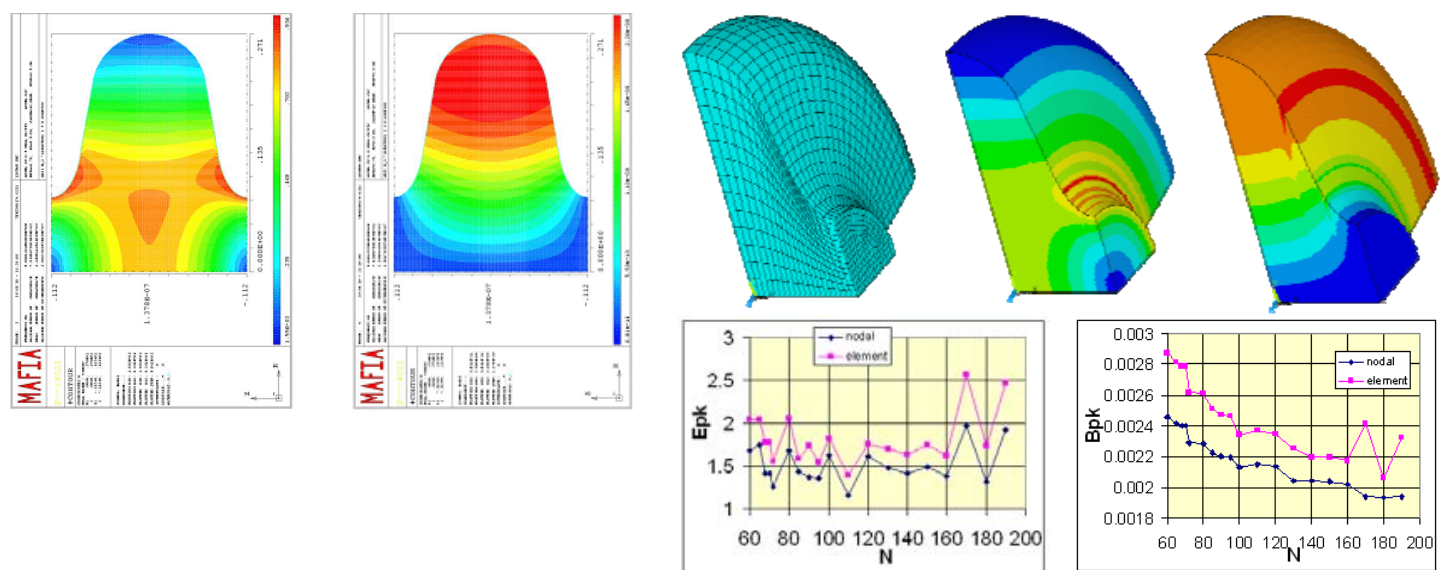

Figure 2: Single-cell elliptic cavity simulations with regular mesh (left - MAFIA simulations).
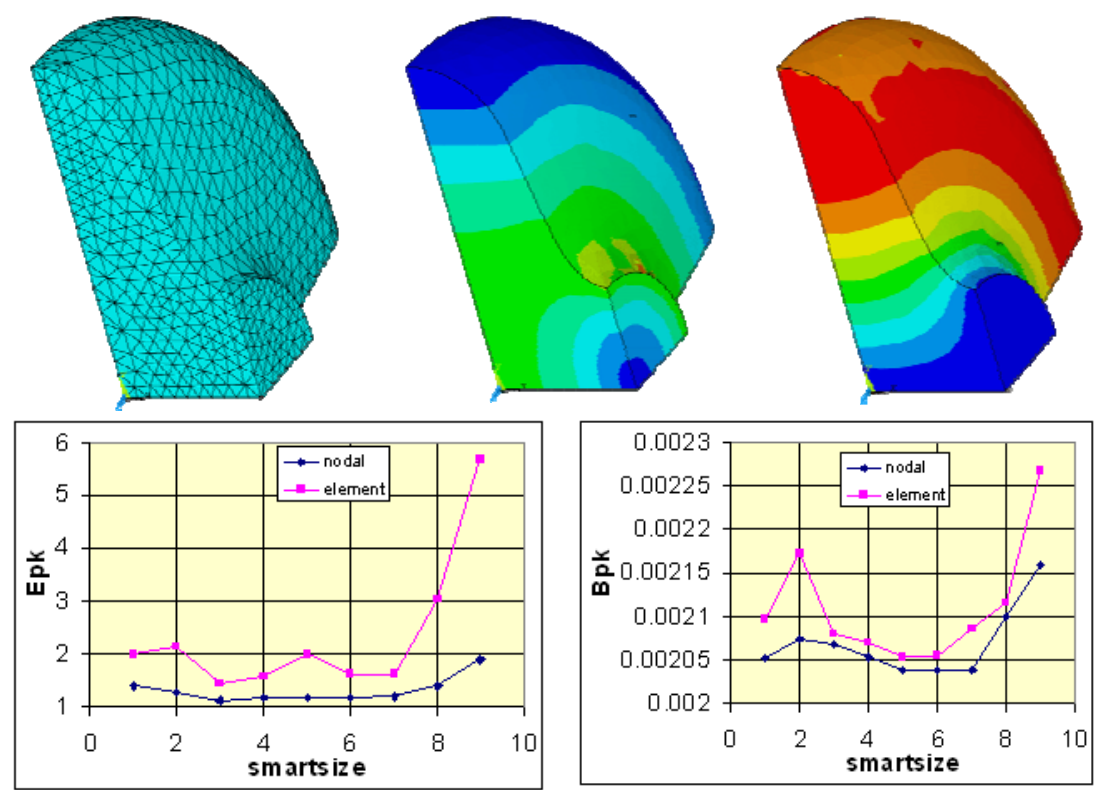

Figure 3: Single-cell elliptic cavity simulations with auto mesh generation (lower "smart size" corresponds more dense mesh).

This could be eliminated using the auto mesh generation again searching for the best mesh tense (Fig.3). As a rule, only a combination of all possible mesh generation tool adjustments can bring the proper result (Fig.4). 
The final mesh adjustment should be provided taking into account the real field distribution in the cavity. Since in Half-Wave Resonator the magnetic field distribution is not symmetric between inner and outer electrodes the optimal mesh is also should be not symmetric. Fig.5 shows the results of the mesh tune in FZJ HWR. Here every model differs by only one mesh step at the cavity dome.
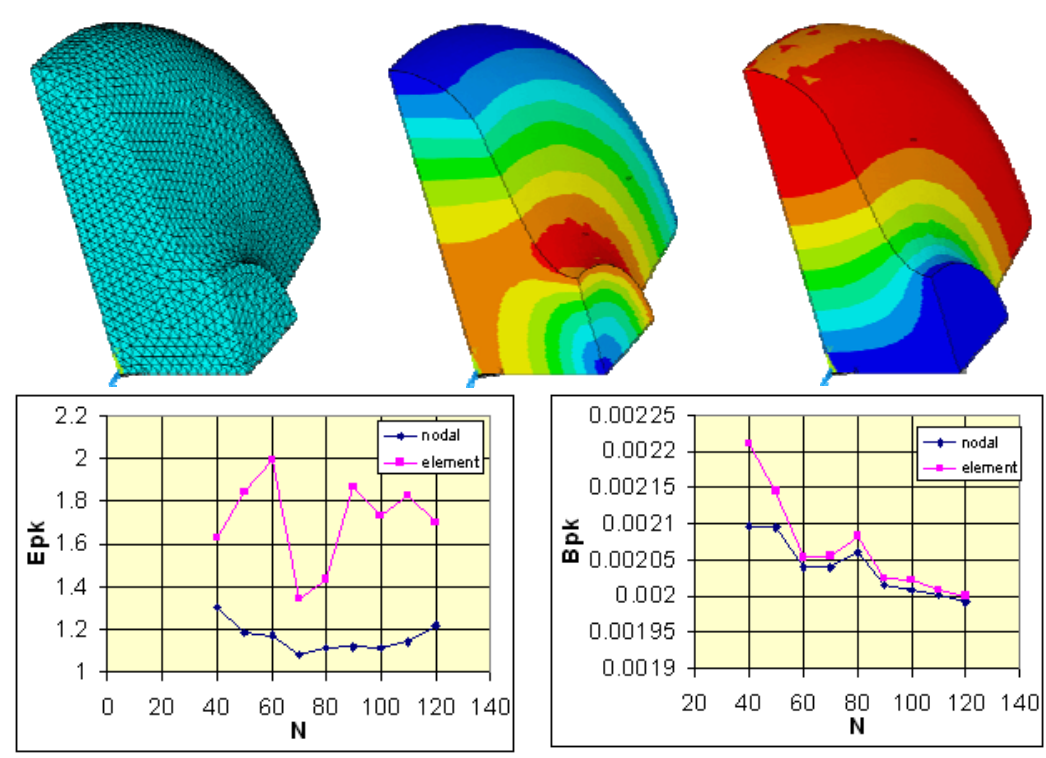

Figure 4: Single-cell elliptic cavity simulations with optimised auto mesh generation.

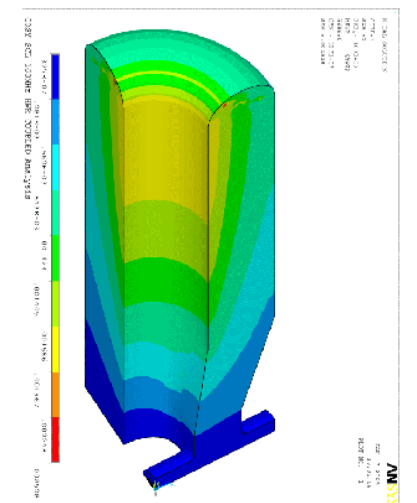

$\mathrm{H}_{\mathrm{pk}}=0.002528$

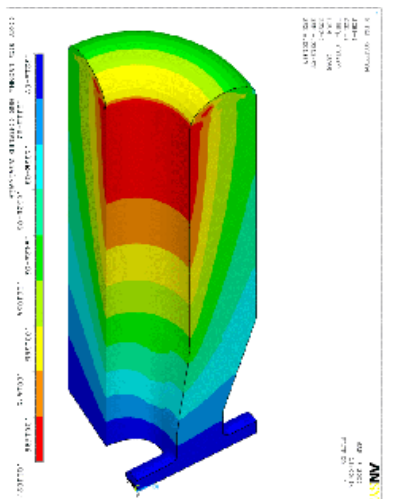

$\mathrm{H}_{\mathrm{pk}}=0.001897$

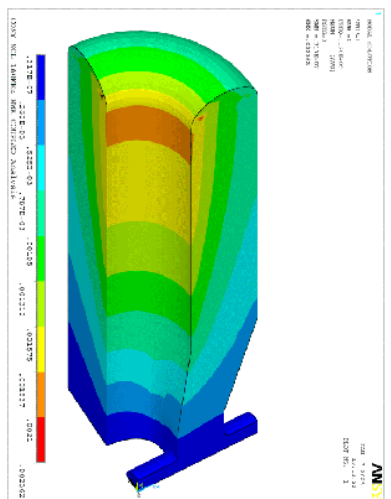

$\mathrm{H}_{\mathrm{pk}}=0.002362$

Figure 5: Half-Wave Resonator magnetic field distribution with different mesh around $\mathrm{B}_{\mathrm{pk}}$ region.

The most complicated case for the optimal mesh generation if the location of $E_{\mathrm{pk}}$ and $\mathrm{B}_{\mathrm{pk}}$ in the cavity changes the position during cavity design like in triple-spoke cavity (Fig.6, the dashed line on the plot indicates the moment of $\mathrm{E}_{\mathrm{pk}}$ location change). 


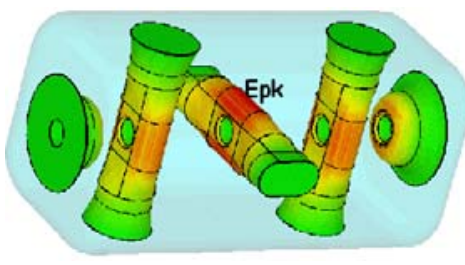

zbar/zcell $=0.3$

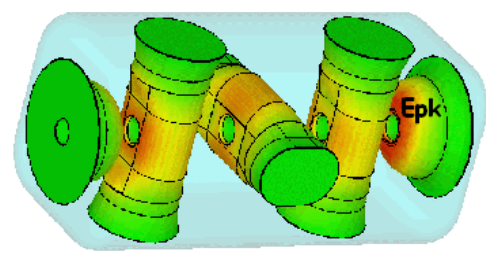

zbar/zcell $=0.53$

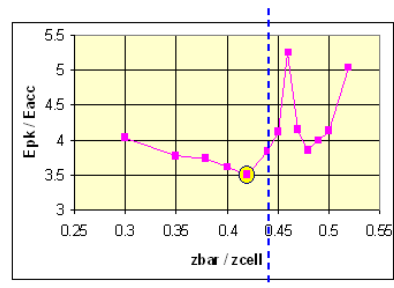

Figure 6: $\mathrm{E}_{\mathrm{pk}}$ location for different triple-spoke cavity geometries

In this case the use of auto mesh generation (for instance in MWS) can cause wrong field calculation results (like peaks in the field dependence behaviour). The use of the more dense mesh also doesn’t guarantee the better results (Fig.7).
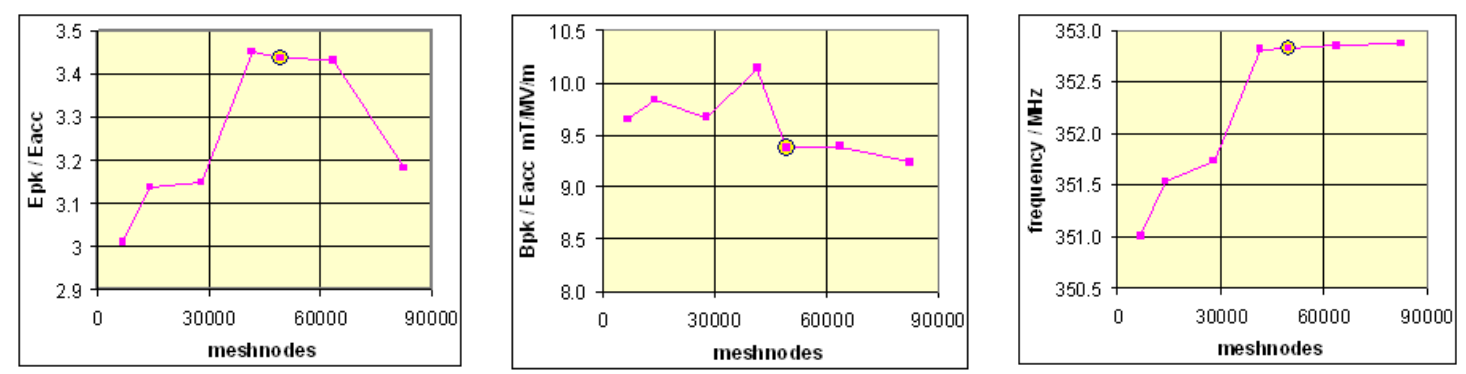

Figure 7: Cavity parameter dependences on mesh dense.
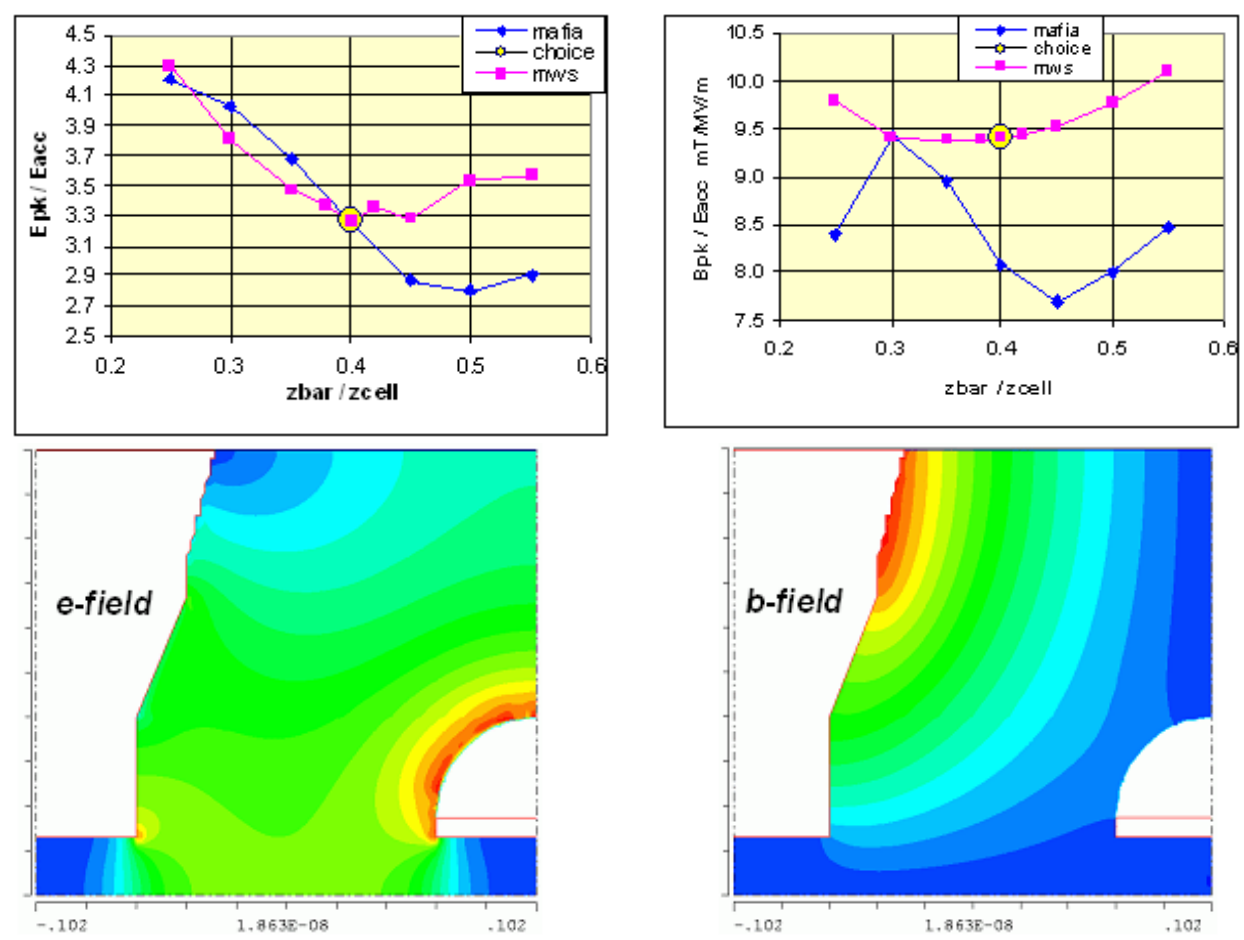

Figure 8: Mesh optimisation in MAFIA 
At the same time the use of the manual mesh generation (like in MAFIA) could produce the controlled mesh in the region of interest and more reliable results (Fig.8, e-field region mesh optimised, b-field region mesh not optimised). It has been noticed that MAFIA usually calculates at least 20\% lower values for peak fields.

\section{Cavity simulations}

All superconducting rf resonators are niobium cavities that are enclosed within helium vessels. These vessels are filled with liquid helium that floods the cavities and maintain the $2 \mathrm{~K}$ operating temperature. Mechanical analysis consist of design calculations for all critical cavity assembly components, cavity tuning sensitivity analysis, active tuner and bench tuner load determination, cavity assembly cooldown analysis, natural frequency and random response analysis, inertia friction weld analysis and critical flaw size calculations.

The high repetition rate like $50 \mathrm{~Hz}$ of an accelerator will require a close look to the mechanical resonances of the cavities. Mechanical resonance's can influence the phase behavior of the cavity during a pulse, which can hardly be compensated by a good control system, even if a lot of additional power is available. Additionally, the cavity RF resonance is sensitive to vibrations of sub- $\mu \mathrm{m}$ amplitudes. These microphonic effects cause low frequency noise in the accelerating fields. Therefore, a careful mechanic eigen mode analysis of the cavity together with its environments should be conducted.

Let's use as an example of the RF resonator structural design a triple-spoke cavity that is under developments in Forschungszentum Juelich (Fig.9). The very first cavity investigation in terms of the mechanical properties is its mechanical eigen modes. We use the criterion of $200 \mathrm{~Hz}$ for the first mode that would be sufficient for the cavity rigidity. To improve the cavity rigidity the stiffening rings on both cavity ends are used (Fig.9). The stiffening rings are $3 \mathrm{~cm}$ high and $2.5 \mathrm{~cm}$ wide. The cavity fixation in four points by every ring is arbitrary and in fact not practical but it allows investigating the modes that related only to the cavity. Here we should notice, as there could not be the absolute cavity RF design, so the structural. Every time one should optimise the structure design in the relation to the cavity future application and real cavity working environments. The results of the experimental mechanical conditions will depend mainly on the cavity fixation in the cryostat.

The lowest cavity mechanical eigen mode is around $261 \mathrm{~Hz}$, which detects the sufficient cavity own rigidity. Without the stiffening ring the first eigen mode frequency lows down as the cavity becomes more flexible in the transverse direction.

The further strategy of such type cavity design should include the integrated simulations of RF and mechanical properties (coupled analyses). The main idea and advantage of the coupled analyses (CA) with numerical codes like ANSYS is to use the same meshed model through all kind of simulations. Such CA in our case is helpful for cavity resonance frequency change calculations caused by different 
mechanical loads. The use of the same mesh during simulations or later the same but deformed mesh should increase an accuracy of the results

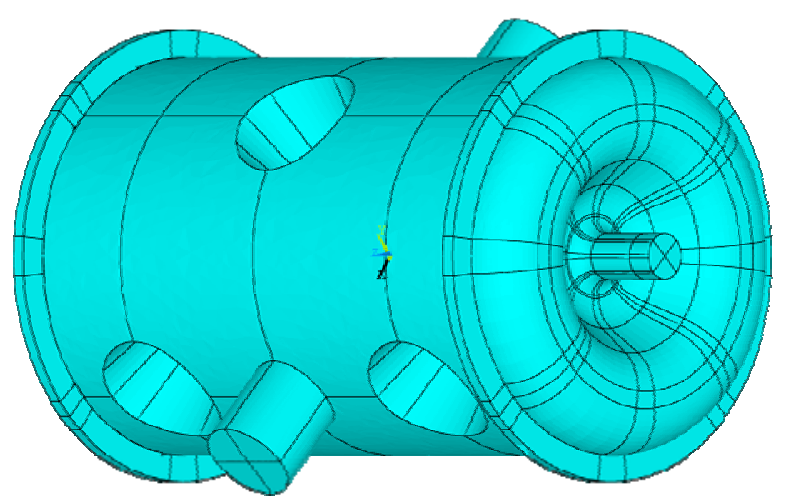

\begin{tabular}{|c|c|c|}
\hline Mode & $\begin{array}{c}\text { w. } \\
\text { ring }\end{array}$ & $\begin{array}{c}\text { no } \\
\text { Freq / } \\
\text { ring }\end{array}$ \\
$\begin{array}{c}\text { Freq / } \\
\mathrm{Hz}\end{array}$ \\
\hline 1 & 261 & 155.1 \\
\hline 2 & 294.5 & 251.2 \\
\hline 3 & 298.6 & 253 \\
\hline 4 & 298.7 & 274.8 \\
\hline 5 & 418.2 & 353.7 \\
\hline
\end{tabular}

Figure 9: Triple-spoke cavity with stiffening ring and cavity eigen modes with unstiffened end-caps.

Let's apply the tuning pressure to the cavity walls close to the central spoke where the magnetic field is close to its maximal value. The slow cavity tuning is required for the cooldown-to-cooldown difference compensation and 1 bar pressure frequency shift compensation. Our simulations result in $75 \mathrm{kHz} / \mathrm{mm}$ tuning sensitivity for the relatively local pressure application (just in three nodes, Fig.10). If required, the tuning zone can be made larger. There are advantages to install the tuner in this place rather as usual at the cavity ends - first, one saves space between the cavities that reduces accelerator length, the second, the required tuning force is much lower as it shouldn't deform the stiffened end cup but only 3-4 mm thick Nb wall. Disadvantage is that the tuning sensitivity is lower.

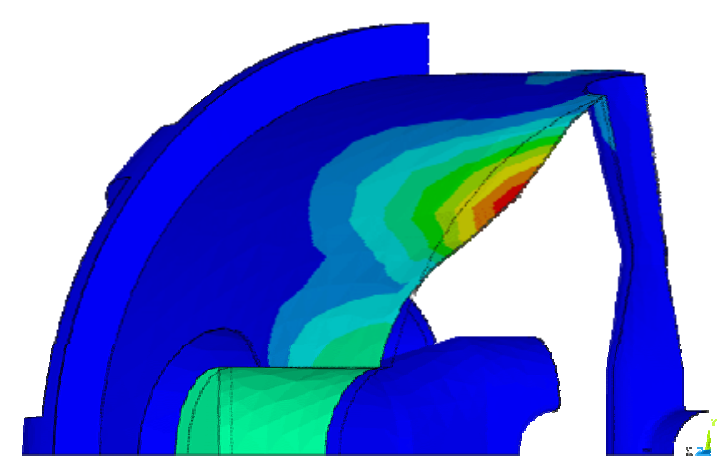

Figure 10: Option of triple-spoke cavity tuning by the mid-spoke (only $1 / 8^{\text {th }}$ of geometry is shown).

The most flexible parts of the structure are the cavity end cups. As soon as the tuner is moved to the middle of the cavity, the end cups can be stiffened as much as it is required. 

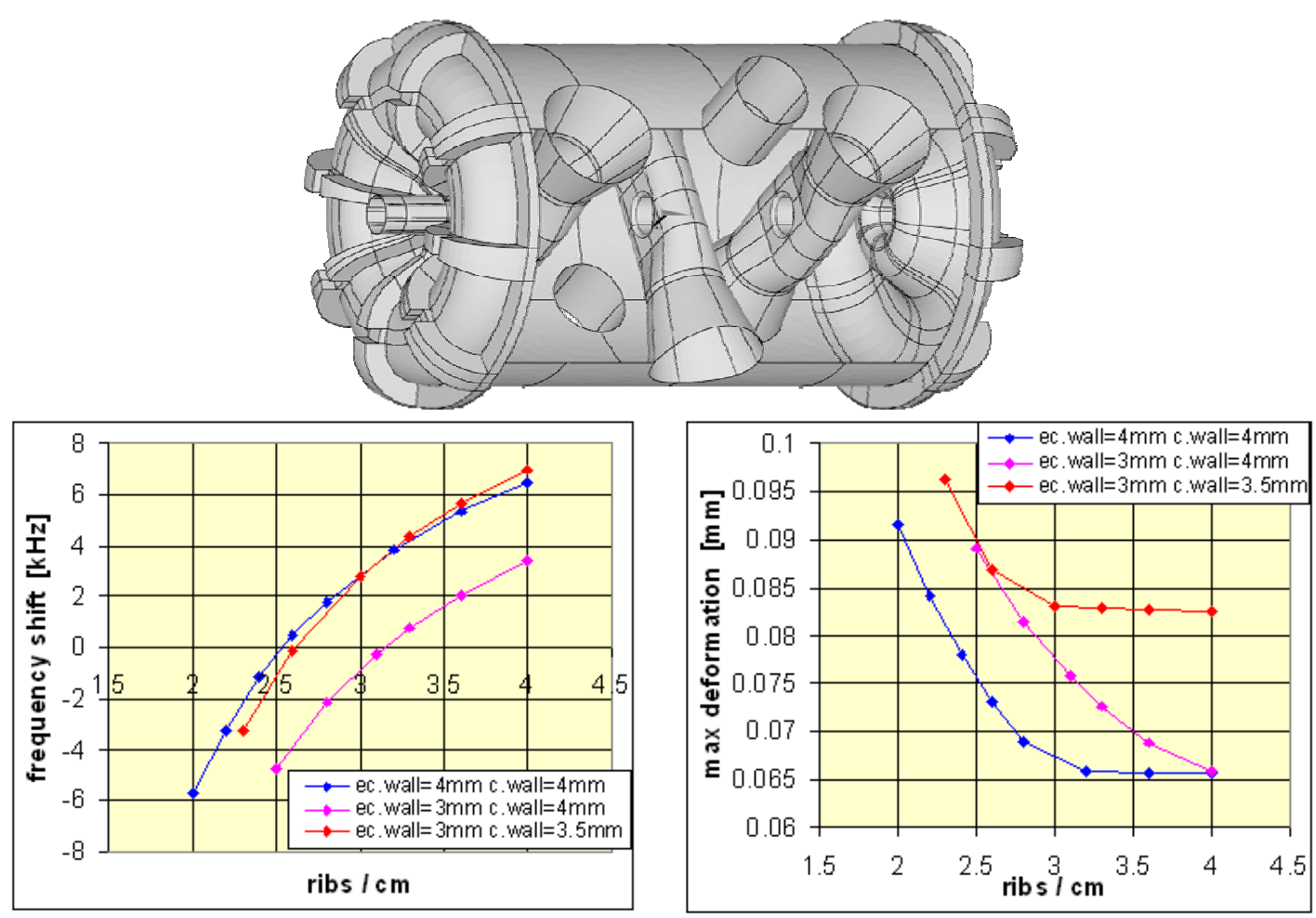

Figure 11: Triple-spoke cavity with stiffening end cup ribs and cavity reaction on 1 bar external loads vs. rib heights.

Let's consider the option with additional end cup stiffening ribs (Fig.11). Here the rings are $4 \mathrm{~cm}$ high and $2.5 \mathrm{~cm}$ wide. The ribs are $10 \mathrm{deg}$. wide, which should automatically, follows the end cup surface increase with radius. It makes end cup rigidity increase with radius.

If to suppose that the end cup doesn't move at all, the frequency shift from 1 bar pressure would be defined mainly by the magnetic field volume change (cavity inductance reduction). The end cup displacement caused by 1-bar pressure results in the last gap length reduction (cavity capacitance increase). It means, both effects work in the different direction of the cavity frequency change. Hence, one can find the end cup stiffening that would result in end cup deformation, which would just compensate the frequency change owing to magnetic field volume change. The rib height variation shows this effect with frequency shift crossing the zero (Fig.11). An uncertain factor here is the end cup wall thickness. We investigated two different cases for end cup wall thickness ( 3 and $4 \mathrm{~mm}$, only for end cup, the rest cavity including spokes is $4 \mathrm{~mm}$ ). One can see on pictures that the maximum deformation moves from end cup to the cavity wall. It is impossible a priory to predict the final wall thickness. That's why one should foresee the possibility to adjust the structure to the final conditions. One-way is to build the ribs as high as possible and after initial measurements to cut them in the region of the main deformations making them less rigid. This method is rather close to the conducted simulation of the rib height variation. The other way is to make the deep and short cut in the same region. Most probably the both methods can be applied. 
The uncertainty with the cavity wall thickness changes the results of the structure optimisations. This once again makes the possibility of the final structure adjustment highly necessary. The use of the tuner in the place of the maximum magnetic field means the complete (again, in the certain extend) fixation of the wall in this place. This results in the lower frequency shift caused by magnetic field change and as a result „overcompensation“ by the end cup displacement. In this case to reach the complete compensation one should either provide bigger end cup stiffening or one could use the tuner as an additional tool for cavity frequency shift adjustment. Here (Fig.10) the case when all four places around spoke are connected to the tuner and fixed is shown. In practice, the slow tuner will be connected only in two places. At the same time that would be also the best place for the fast tuner. It means, at least three of four places will be fixed.

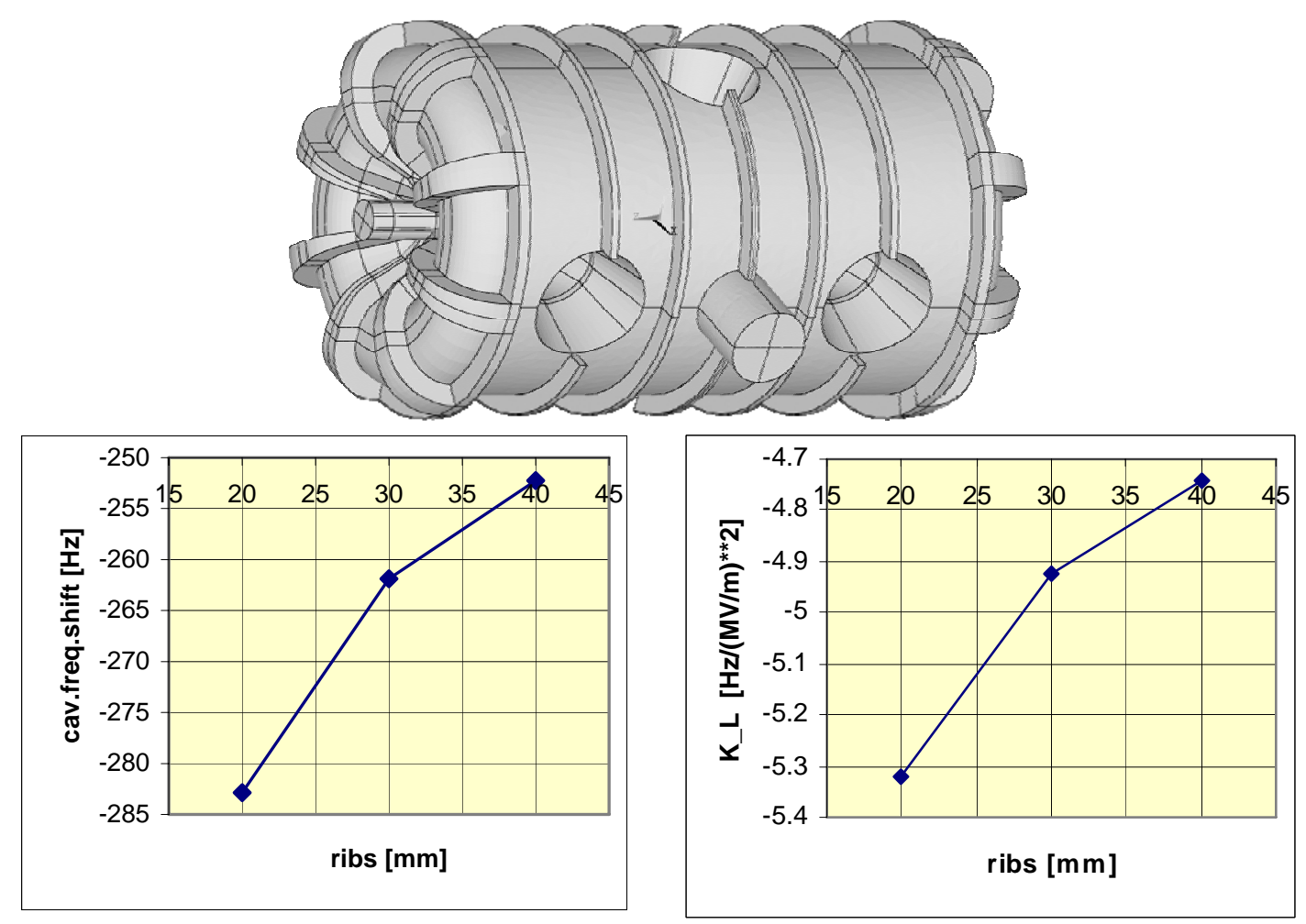

Figure 12: Triple-spoke cavity with stiffened end cup and Lorenz force ribs and cavity reaction on Lorenz forces vs. rib heights.

Lorentz force cavity detuning is a function of RF field, which is forcing term, mechanical mode frequency, modal mass and mode's damping degree. However, findings of mode frequencies, corresponding stiffness and especially damping degrees are quite difficult for the real situation, since these dynamic properties are very sensitive to the boundary conditions such as connection scheme, strength, equivalent masses and equivalent stiffness of surroundings that is attached to the cavity. Only the relative comparisons are available before having experimental measurements of mechanical properties with actual cryo-module. Even after having 
measured values about dynamic mechanical properties of cavity, the predictions sometimes are not accurate with a conventional RF modeling, since RF fields and mechanical vibrations are strongly coupled and both are dynamic.

The measure of the static Lorenz force cavity detuning is a structure frequency shift relative to the accelerating cavity field square $\left(K_{L}=\Delta f /\left(E_{a c c}\right)^{2}\right)$. The action of the Lorenz forces in the region of the magnetic field is directed outside, which is quite opposite to the action of the 1-bar pressure. It means the addition of effects of cavity wall and end cup deformations. In this case the use of the tuners (say, cavity wall fixation in these places) reduces Lorenz force cavity detuning.

For further LFD reduction one could use small ribs $(1 \times 2.5 \mathrm{cmxcm})$ on the cavity walls in the regions of maximal deformations (Fig.12). LFD rib installations will also affect the cavity frequency shift caused by 1-bar pressure. It results in stronger „overcompensated“ case. Anyway, LFD ribs should be installed as the tuner will compensate the bigger LHe pressure detuning and LFD will be lower. Let's finally note, that investigations and optimizations of all effects are within $0.1 \mathrm{~mm}$, which means strong uncertainties between simulations and the final cavity geometry as the tolerances on cavity manufacturing are within $0.2 \mathrm{~mm}$. Nevertheless, the final practical cavity adjustment is possible in the case of the methods of fine-tuning included in the cavity design beforehand. With a use of LFD ribs the dependence on the cavity wall thickness for the frequency shift is negligible.

\section{Summary}

There is a possibility to use combined analytical-numerical calculations. The simple cell shape of the elliptic cavity allows using effectively the perturbation method for single cell Lorenz force detuning (LFD) calculations (Fig.13). For this cavity we used 2D electromagnetic fields and Lorenz force pressure calculated with MAFIA, transferred the pressure into ANSYS 2D model, evaluated the structure displacements and calculated LFD. The results are summarized in Table 1. Still, LFD measurements can differ much from one measurement to another since they are most sensitive to the cavity constrains in the cryostat. Additionally, the cavity displacements caused by Lorentz forces are within $\mu \mathrm{m}$ 's, and the cavity manufacture tolerances usually 100-200 $\mu \mathrm{m}$. That's why one should expect here the biggest difference between simulation and experimental data. The cavity constrains in the cryostat should be simulated as close to the reality as possible since they can play the dominant role for accuracy of the simulation results.

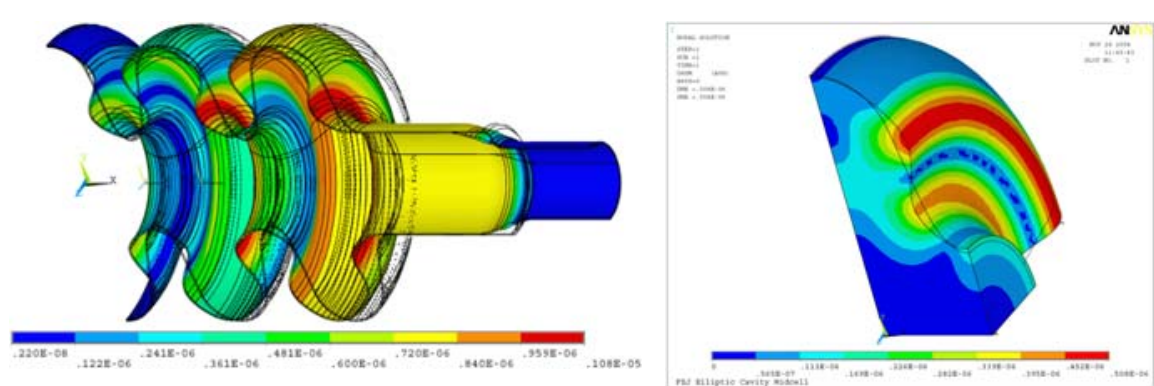

Figure 13: Elliptic cavity deformations by Lorenz forces 


\begin{tabular}{|c|c|c|c|c|}
\hline LFD & 5-cell & single & exp & single/pert.m. \\
\hline $\mathrm{K}_{\mathrm{L}}\left[\mathrm{Hz} /(\mathrm{MV} / \mathrm{m})^{* *} 2\right]$ & -3.08 & -2.99 & -3.72 & -3.95 \\
\hline
\end{tabular}

Table 1: Elliptic cavity static Lorenz force detuning simulations.

The good agreement has been detected during relative crosscheck with ANSYS codes of different simulations and their combination (Table 2).

\begin{tabular}{|l|c|c|c|}
\hline & max displ. & df & $\mathrm{K}_{\mathrm{L}}$ \\
\hline & $\mathrm{mm}$ & {$[\mathrm{Hz}]$} & $\mathrm{Hz} /(\mathrm{MV} / \mathrm{m})^{* * 2}$ \\
\hline 1 Bar & 0.1 & -17698.7 & \\
\hline LFD+1bar & 0.101 & -17944.9 & \\
\hline (LFD+1bar)-1Bar & 0.001 & -246.22 & -3.8472 \\
\hline LFD & .000994 & -243.86 & -3.8103 \\
\hline
\end{tabular}

Table 2: HWR data comparison.

\begin{tabular}{|l|l|c|r|r|r|}
\hline \multicolumn{2}{|c|}{} & $\begin{array}{c}\text { Freq. } \\
{[\mathrm{MHz}]}\end{array}$ & $\begin{array}{c}\text { df_calc. } \\
{[\mathrm{kHz}]}\end{array}$ & $\begin{array}{c}\text { df / f } \\
\%\end{array}$ & $\begin{array}{r}\text { df_exp. } \\
{[\mathrm{kHz}]}\end{array}$ \\
\hline elliptic & $\begin{array}{l}\text { cool- } \\
\text { down }\end{array}$ & 500 & 1500 & 0.3000 & \\
\cline { 2 - 6 } & 1 Bar & 500 & 5.73 & 0.0110 & \\
\cline { 2 - 6 } & LFD & 500 & 0.6 & 0.0001 & 0.73 \\
\hline HWR & $\begin{array}{l}\text { cool- } \\
\text { down }\end{array}$ & 160 & 370 & 0.2313 & 260 \\
\cline { 2 - 6 } & 1 Bar & 160 & 30 & 0.0188 & 50 \\
\cline { 2 - 6 } & LFD & 160 & 0.3 & 0.0002 & 0.25 \\
\hline spoke & $\begin{array}{l}\text { cool- } \\
\text { down }\end{array}$ & 356 & 755 & 0.212 & \\
\cline { 2 - 6 } & 1 Bar & 356 & 379 & 0.106 & 440 \\
\cline { 2 - 6 } & LFD & 356 & 2.22 & 0.0006 & 2.25 \\
\hline \multirow{2}{*}{$\begin{array}{l}\text { triple- } \\
\text { spoke }\end{array}$} & $\begin{array}{l}\text { cool- } \\
\text { down }\end{array}$ & 760 & 1549 & 0.2038 & 1280 \\
\cline { 2 - 6 } & 1 Bar & 760 & 618 & 0.0813 & 500 \\
\cline { 2 - 6 } & LFD & 760 & 5 & 0.0007 & 5.32 \\
\hline
\end{tabular}

Table 3: Cavity frequency shifts.

Table 3 summarizes the calculated and measured frequency shifts of different cavities [4]. The big cavities with lower frequencies (HWR in our case) can be simulated with better accuracy since all deformations are relatively small to compare with cavity size and will cause lower relative frequency drifts. The cavity cool-down to $2 \mathrm{~K}$ results in the cavity contraction with the biggest structure deformations and hence, the largest mesh displacements. The result accuracies of these simulations are mostly defined by mesh dependence error. 


\section{Acknowledgements}

We acknowledge the support of the European Community-Research Infrastructure Activity under the FP6 "Structuring the European Research Area" programme (CARE, contract number RII3-CT-2003-506395)

\section{References}

[1] MAFIA, MWS are trademarks of CST Inc. www.cst.com.

[2] HFSS is a trademark of ANSOFT Inc. www.ansoft.com.

[3] ANSYS is a trademark of SAS Inc. www.ansys.com.

[4] E. Zaplatin, "FZJ SC Cavity coupled analyses", Workshop on RF Superconductivity SRF06, 2005, Ithaca, USA. 